\title{
Partially Blocked Balanced Study
}

National Cancer Institute

\section{Source}

National Cancer Institute. Partially Blocked Balanced Study. NCI Thesaurus. Code C90432.

A type of randomized clinical trial in which study subjects are arranged into groups with equal numbers by prespecified criteria, such as race or sex, to reduce variability of results within a treatment group. 